\title{
Clinical Research Training During Gastroenterology Fellowship
}

\author{
Saad Saffo ${ }^{1} \cdot$ Tamar Taddei $^{1,2}$ \\ Accepted: 8 January 2022 / Published online: 3 February 2022 \\ (c) The Author(s), under exclusive licence to Springer Science+Business Media, LLC, part of Springer Nature 2022
}

\section{Introduction-Training Pathways During Gastroenterology Fellowship}

Gastroenterology (GI) fellowship is clinically rigorous. First-year fellows across the USA face that reality each July as they begin to navigate a diverse curriculum and steep learning curves to acquire the knowledge base, procedural skills, and efficiency necessary to manage patients with a multitude of gastrointestinal and hepatobiliary diseases. Despite the intense clinical burden, involvement in research is also an integral part of fellowship. The clinical experiences fellows have early in their training often translate into creative and practical research projects during the latter part of fellowship, when most have the opportunity to pursue scholarly activities. Although this time is often variably allotted by training programs that lack external funding, some trainees are supported by federal grants through the National Institutes of Health (NIH) such as the T32 Institutional National Research Service Award (NRSA). These grants permit fellows to have protected time and access to scholarly activities and mentorship to develop the knowledge base and skill set necessary to have a successful career in clinical investigation.

The T32 NRSA is currently available to trainees in 26 $(12.5 \%)$ of the 208 adult GI fellowships in the USA [1]. Fellows who receive T32 support typically devote most of their final two years of fellowship toward research training, with the exception of weekly continuity clinics and other clinical activities needed in order to ensure compliance with American Board of Internal Medicine (ABIM) requirements [2]. Although the timing of clinical and research periods may vary within the fellowship cycle for each individual,

Saad Saffo

saad.saffo@yale.edu

1 Section of Digestive Diseases, Department of Internal Medicine, Yale University School of Medicine, 333 Cedar Street, 1080 LMP, New Haven, CT 06520-8019, USA

2 West Haven Veteran Affairs Medical Center, West Haven, CT, USA classically, trainees begin their research time after some period of intensive clinical training and are encouraged to minimize disruptions such as clinical duties and moonlighting while engaging in research activities. Specifically, the NIH stipulates that trainees should dedicate $40 \mathrm{~h}$ per week to research training and "may spend on average, an additional $25 \%$ of their time (e.g., $10 \mathrm{~h}$ per week) in part-time research, teaching, or clinical employment, so long as those activities do not interfere with, or lengthen, the duration their NRSA training" [3].

In addition to the federal guidelines that accompany T32 grants, most institutions provide a roadmap to guide both trainees and mentors. Regardless of whether fellows receive support from their institution alone or have additional funding from the $\mathrm{NIH}$, research experiences often vary due to a variety of individual and institution-related factors, thus raising questions about the aims and implementation of research activities during fellowship. Here, we discuss: (1) the core components of research training; (2) how individuals and training programs can optimize the experience; and (3) the overarching goals. We address these issues by proposing a basic framework for clinical research training for current and prospective GI fellows.

\section{The Building Blocks-Core Components of Research Training}

The core components of research training during fellowship include education in research methodology, mentorship, and completion of an original research project. Study design, statistics, and bioethics are some aspects of research methodology that may be learned through formal in-person (or virtual) classroom-based didactics or self-directed education, often supplemented by online-based modules and lectures. Many fellowship programs allow trainees to formalize this process by pursuing additional degrees or certificates in health sciences research, and in some instances, the cost of an additional degree may be partially offset by the T32 grant (currently up to $\$ 16,000$ per year) [3]. 
Next, although mentorship is an essential aspect of research training, this is anecdotally the most variable feature. The spectrum of mentorship is broad: Some fellows seek or receive limited or scattered mentorship, whereas others may have sustained and structured mentorship from several faculty members within or outside their department or institution. At a minimum, trainees supported by a T32 grant must have at least one designated mentor or sponsor, typically an established investigator, overseeing their progress. Although there is limited objective literature addressing the benefits of having structured mentorship, institutions that cultivate a culture of mentorship may have improved success in attaining future federal funding [4]. For most trainees, it can be helpful to identify personal, career, and skill/content mentors with different objectives for each mentor-mentee relationship, although it is also reasonable to have the same mentor serve in more than one capacity in some cases. The frequency of meetings and the type of mentorship can be tailored to the situation (i.e., focusing on personal or skill mentoring in the early stages of fellowship then prioritizing career mentoring in the middle or late stages).

Finally, designing, implementing, and communicating an original high-impact study are principal goals of the research training experience. Although the aim of fellowship is largely to develop the skill set and framework for future progress, it is reasonable to achieve some balance between educational activities and research productivity. In most cases, completing at least one study from conception through publication is a feasible goal that also may serve as a foundation for future grant proposals. Often, these represent well-designed retrospective studies that incorporate data from chart reviews or administrative databases, although some trainees are able to complete prospective studies. Having a study advisory committee of around five faculty members with complementary areas of expertise (e.g., basic sciences, clinical research, clinical care, statistics, bioinformatics) from within and outside of the GI section helps provide the necessary support, balance, and oversight for the project. Publishing the findings in the form of a manuscript is highly informative for trainees, providing hands-on experiences in scientific writing and the revision and submission processes. The overall progress of T32 recipients is often included in a Research Performance Progress Report (RPPR) that is submitted by the section to the NIH/National Institute of Diabetes and Digestive and Kidney Diseases (NIDDK) on an annual basis [5].

\section{Optimizing the Experience-Opportunities and Distractions}

Beyond NIH and institutional guidelines and the core components of research training, fellows often have flexibility in assessing which activities represent opportunities for growth and scholarly achievement and which represent possible distractions, albeit the distinction is not always entirely apparent. Although a number of topics can be potentially discussed, the authors would like to focus on three in particular: collaborative research groups, additional degrees, and endoscopic training.

\section{Collaborative Research Groups}

Aside from mentors and study/thesis committees, collaborative research groups may represent a productive way to share research ideas and experiences, provide professional advice, develop collaborations, and learn and teach new methods. They can include students, residents, and fellows at different stages of training and may also include faculty mentors or other research staff. In contrast to other types of mentorship, these groups enable trainees to learn from and teach one another, fostering a spirit of collaboration and encouraging more senior fellows to take on mentorship of junior trainees. During sessions, trainees can present original ideas or research in progress to their peers in a less stressful environment in order to gain general feedback and assistance with analytical questions, among other things. Nevertheless, over-involvement in group activities may also have negative consequences since trainees may become sidetracked with unrelated research projects or participate in excessive or extraneous meetings and seminars, which when compounded with general fellowship conferences and additional mentorship and committee meetings, may be unduly burdensome.

\section{Additional Degrees}

As previously mentioned, some $\mathrm{T} 32$ grant recipients may wish to pursue additional masters or doctoral degrees in health sciences research during their training. Although not required in most cases, these degrees often provide validation of content mastery and exemplify dedication to research that could help trainees in future efforts to secure grant funding and attain desired employment and promotions. On the other hand, the additional course work and degree requirements may have the potential to detract to some extent from research productivity and involvement in other types of highyield academic or clinical activities. Furthermore, since the content of some required courses may not be individualized to the needs of each trainee, significant time could be spent learning content or skill sets that may not be directly applicable to their research interests. For some fellows, working toward a clinical research certificate may represent an efficient and cost-effective alternative to a masters or doctoral degree, although the decision ultimately depends on each trainee's time constraints and future goals. Mentor guidance with this decision making can be helpful with weighing available options. 


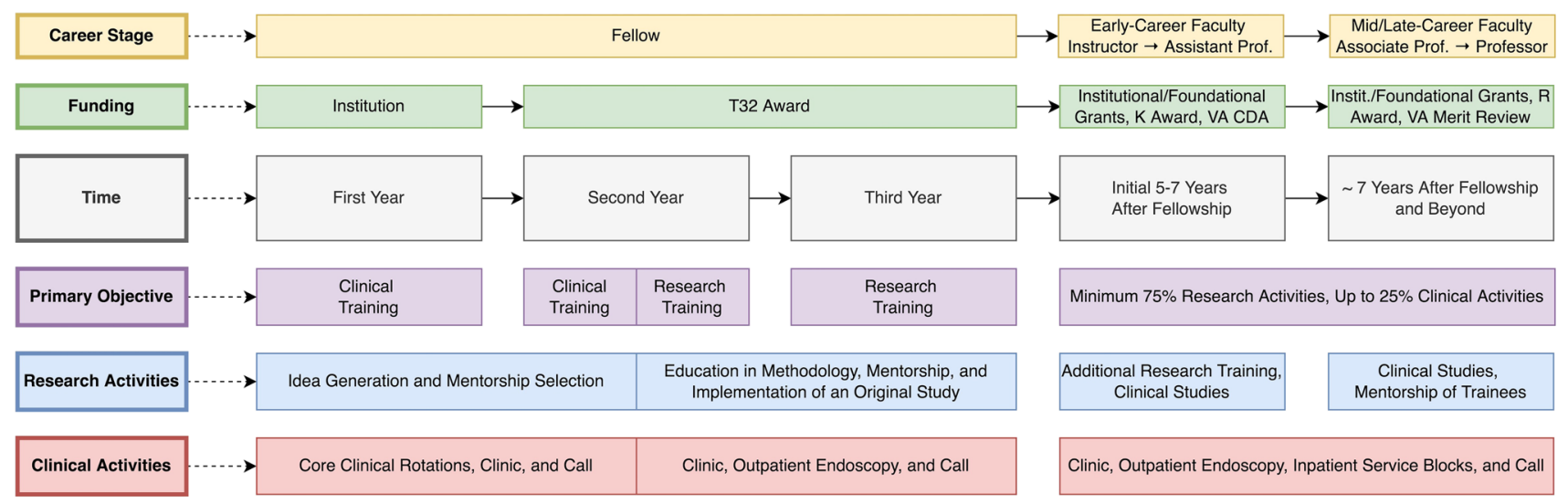

Fig. 1 Timeline for gastroenterology fellows pursuing a career in clinical research

\section{Endoscopic Training}

A fundamental component of fellowship for all GI fellows is endoscopic training. Though first-year fellows develop basic endoscopic techniques, skills are usually refined with attention to the nuances of more advanced procedures such as polypectomy/endoscopic mucosal resection during the second and third years of training. During this period, endoscopy time may be determined in part by fellows themselves and dependent upon their comfort level and procedural volume, among other factors. Some fellows may have a tendency to devote excess time to endoscopy as the increasing familiarity with procedures may prevail over the more difficult and nebulous task of learning new research skills, whereas others may choose to considerably limit endoscopy time in order to concentrate on their scientific endeavors. Although endoscopy may represent a relative distraction to research productivity, adequate exposure to endoscopy should be encouraged since ample procedural training is an essential aspect of GI fellowship. Approximately one half day per week is reasonable for most individuals during protected research time, although trainees should seek guidance from their mentors so as to determine the appropriate amount of time that should be devoted to endoscopy on a case-bycase basis. For fellows interested in endoscopic research, the time spent in the endoscopy suite may also help facilitate research efforts by allowing them to formulate new research questions and recruit patients for studies. At a minimum, the American Society of Gastrointestinal Endoscopy (ASGE) recommends that 275 colonoscopies and 130 esophagogastroduodenoscopies are necessary in order to achieve competency, requirements that have been adopted by the Accreditation Council for Graduate Medical Education (ACGME) [6].

\section{Fellowship and Beyond-Career Goals}

The overall goal of the T32 grant is to prepare "trainees for careers that have a significant impact on the health-related research needs of the Nation" [7]. Ideally, the research experiences during fellowship will pave the way for an NIH Research Career Development (K) Award or Veterans Affairs (VA) Career Development Award (CDA), which typically provide mentored funding for up to 5 years. The application for these grants may be submitted during the final year of fellowship, although some candidates may require additional training prior to submitting a successful grant application (Fig. 1). At the conclusion of the K or CDA award, individuals may apply for a research (NIH R-series or VA Merit Review) grant to support independent research [8].

Currently, though there is no published information detailing the career paths of GI T32 grant recipients, the overall data for all T32 recipients from 1995 to 2009 $(N=35,528)$ followed through 2017 were recently published by the NIH [9]. Notably, 6923 individuals (19.5\%) applied for K awards, of whom 4236 (61.2\%) received funding, and 8294 individuals (23.3\%) applied for R awards, of whom 4299 (51.8\%) received funding. Analysis of these data suggests that a number of demographic variables (younger age, male sex, and $\mathrm{PhD}$ level of education) were associated with an increased likelihood for a trainee to apply for and receive an R award.

Although research success is often generally measured by the awarding of K, R, CDA, and Merit Review grants, many can achieve their career goals without formal NIH or VA funding. Ultimately, fellows will have to make individual decisions about the role of research in their careers, and some may choose predominantly clinically focused careers with limited or no research involvement. Others may continue to perform focused clinical research, sometimes relying 
on institutional or foundation grants and often using their own administrative time to support these efforts, though this becomes increasingly challenging in the context of mounting clinical responsibilities. Regardless, the research skills acquired during fellowship will positively impact trainees during the course of their careers. As the scientific body of literature grows and clinical practice evolves with increasing emphasis on personalized medicine, future gastroenterologists will need to be prepared to apply their knowledge in clinical research to understand practice-changing studies and direct individualized patient care.

Acknowledgments The authors would like to acknowledge Dr. Michael $\mathrm{H}$. Nathanson $\mathrm{MD} / \mathrm{PhD}$ for his assistance with this manuscript.

Funding Saad Saffo is funded by NIH T32 2T32DK007356-42.

\section{Declarations}

Conflict of interest The authors declare that they have no conflict of interest.

\section{References}

1. Institutional Training Program Locations, National Institute of Diabetes and Digestive and Kidney Diseases. https://www.niddk. nih.gov/research-funding/training-career-development/institutio nal-training-program-locations. Accessed 16 Oct 2021.
2. Gastroenterology Policies, American Board of Internal Medicine. https://www.abim.org/certification/policies/internal-medicinesubspecialty-policies/gastroenterology/. Accessed 16 Oct 2021.

3. NIH Grants Policy Statement: Ruth L. Kirschstein National Research Service Awards, National Institutes of Health. https:// grants.nih.gov/grants/policy/nihgps/HTML5/section_11/11_ ruth_1_kirschstein_national_research_service_awards.htm. Accessed 16 Oct 2021.

4. Choi AMK, Moon JE, Steinecke A et al. Developing a culture of mentorship to strengthen academic medical centers. Acad Med. 2019;94:630-633.

5. Research Performance Progress Report (RPPR), National Institutes of Health. https://grants.nih.gov/grants/rppr/index.htm. Accessed 16 Oct 2021.

6. ASGE Standards of Practice Committee. Guidelines for privileging, credentialing, and proctoring to perform GI endoscopy. Gastrointest Endosc. 2017;85:273-281.

7. Institutional Training Grants, National Institutes of Health. https:// researchtraining.nih.gov/programs/training-grants/t32. Accessed 16 Oct 2021.

8. Berg KM, Gill TM, Brown AF et al. Demystifying the NIH grant application process. J Gen Intern Med. 2007;22:1587-1595.

9. Lauer M. NIH R01 Grant outcomes of T32 postdoctoral participants: associations with demographics, cohort, degree, and career development (K) awards. National Institutes of Health. https:// nexus.od.nih.gov/all/2021/02/01/nih-r01-grant-outcomes-of-t32postdoctoral-participants-associations-with-demographics-cohortdegree-and-career-development-k-awards/. Accessed 16 Oct 2021.

Publisher's Note Springer Nature remains neutral with regard to jurisdictional claims in published maps and institutional affiliations. 